\title{
Theoretical and Numerical Analysis of 1 : 1 Main Parametric Resonance of Stayed Cable Considering Cable-Beam Coupling
}

\author{
Li-Na Zhang, ${ }^{1}$ Feng-Chen Li, ${ }^{2}$ Xiao-Yong Wang, ${ }^{3}$ and Peng-Fei Cui ${ }^{4}$ \\ ${ }^{1}$ School of Transportation and Civil Engineering \& Architecture, Foshan University, Foshan, Guangdong 528000, China \\ ${ }^{2}$ Faculty of Civil and Architectural Engineering, East China University of Technology, Nanchang, Jiangxi 330013, China \\ ${ }^{3}$ College of Engineering, Department of Architectural Engineering, Kangwon National University, \\ Chuncheon 200-701, Republic of Korea \\ ${ }^{4}$ College of Civil Engineering, Hunan University, Changsha, Hunan 410082, China
}

Correspondence should be addressed to Feng-Chen Li; fcli_1980@163.com

Received 5 October 2016; Accepted 4 December 2016; Published 26 January 2017

Academic Editor: Luigi Nicolais

Copyright (c) 2017 Li-Na Zhang et al. This is an open access article distributed under the Creative Commons Attribution License, which permits unrestricted use, distribution, and reproduction in any medium, provided the original work is properly cited.

For the 1:1 main parametric resonances problems of cable-bridge coupling vibration, a main parametric resonances model considering cable-beam coupling is developed and dimensionless parametric resonances differential equations are derived. The main parametric resonances characteristics are discussed by means of multiscale approximation solution methods. Using an actual cable of cable-stayed bridge project for research object, numerical simulation analysis under a variety of conditions is illustrated. The results show that when the coupling system causes $1: 1$ parametric resonance, nonlinear main parametric resonances in response are unrelated to initial displacement of the cable, but with the increase of deck beam end vertical initial displacement increases, accompanied with a considerable "beat" vibration. When the vertical initial displacement of deck beam end is $10-6 \mathrm{~m}$ order of magnitude or even smaller, "beat" vibration phenomenon of cable and beam appears. Displacement amplitude of the cable is small and considerable amplitude vibration may not occur at this time, only making a slight stable "beat" vibration in the vicinity of the equilibrium position, which is different from $2: 1$ parametric resonance condition of cable-bridge coupling system. Therefore, it is necessary to limit the initial displacement excitation amplitude of beam end and prevent the occurrence of amplitude main parametric excitation resonances.

\section{Introduction}

The excitation of parametric resonance is dependent on time and is used as a parameter in the vibration equation of the system [1-3]. According to whether the amplitude and frequency of the excitation change with time or not, the parametric resonance of the stay cable is divided into two cases: When the quality of deck or tower is far greater than the cable, without considering the effect of cables on the deck or tower, as an ideal incentive, the incentive is not affected by the response and the system vibration is simplified as spring-mass [4] and cable-mass [5-9] model, in which the input energy of the stay cable is from the support point; when the vibration of cables and bridge deck or tower are coupled with each other, the system will be coupled with resonance in initial condition of cable-deck or cable-tower system. Finally, the large vibration of these two can be aroused, and the system vibration is simplified as the [10-12] model of the cable flexible beam.

In this paper, the displacement time history response and amplitude-frequency characteristics of the cable are mainly discussed in the case of the $1: 1$ main parametric resonance in the cable-beam coupling system, the approximate solutions of motion equations are solved by the method of multiple scales, and the results are verified by numerical simulation. However, the $2: 1$ parametric resonance and $1: 2$ and $1: 3$ superharmonic resonance response problems are not the focus of discussion here. 


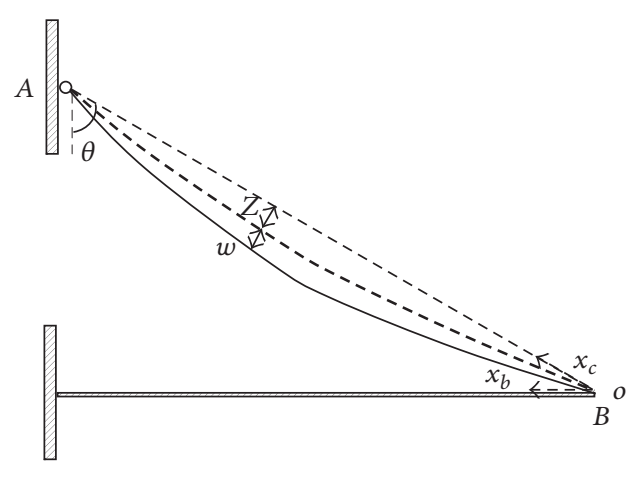

FIGURE 1: Parametric resonance refined model of cable-beam coupling.

\section{Theoretical Model of Parametric Resonance Considering Cable-Beam Coupling}

2.1. Differential Equation of Motion with Parametric Resonance Considering Cable-Beam Coupling. Several basic assumptions are made before establishing the equation of motion for the cable-beam composite structure [13]:

(1) The material nonlinearity of the cable and beam is not considered.

(2) The gravity sag curve of cable is considered as a parabola.

(3) The bending stiffness, torsional stiffness, and shear stiffness of the cable are not considered.

(4) The change of cable force along the length direction is not considered.

(5) Cable is always in elastic state during vibration.

(6) The axial deformation of beam is not considered.

On the premise of the above assumptions, the model is established in Figure 1, considering the refined model of the cable-beam coupling.

The motion trajectory of the cable and the deck beam is described by local coordinate system $o-x_{b} y_{b}$ and $o-x_{c} y_{c}$, respectively, as shown in Figure 1. According to the Hamilton principle, the differential equation of motion for the cablebeam coupling structure is [14]

$$
\begin{aligned}
& m_{c} \frac{\partial^{2} u_{c}\left(x_{c}, t\right)}{\partial t^{2}}+c_{c} \frac{\partial u_{c}\left(x_{c}, t\right)}{\partial t} \\
& =\frac{\partial}{\partial s}\left\{T \frac{\partial\left[u_{c}\left(x_{c}, t\right)+X_{c}\left(x_{c}\right)\right]}{\partial s}\right\}+m_{c} g \cos \theta \\
& m_{c} \frac{\partial^{2} v_{c}\left(x_{c}, t\right)}{\partial t^{2}}+c_{c} \frac{\partial v_{c}\left(x_{c}, t\right)}{\partial t} \\
& =\frac{\partial}{\partial s}\left\{T \frac{\partial\left[v_{c}\left(x_{c}, t\right)+Y_{c}\left(x_{c}\right)\right]}{\partial s}\right\}+m_{c} g \sin \theta \\
& m_{b} \frac{\partial^{2} v_{b}\left(x_{b}, t\right)}{\partial t^{2}}+c_{b} \frac{\partial v_{b}\left(x_{b}, t\right)}{\partial t}+E_{b} I_{b} \frac{\partial^{4} v_{b}\left(x_{b}, t\right)}{\partial x_{b}^{4}}
\end{aligned}
$$

$$
\begin{aligned}
= & \frac{\partial}{\partial x_{b}}\left\{N \frac{\partial\left[v_{b}\left(x_{b}, t\right)+Y_{b}\left(x_{b}\right)\right]}{\partial x_{b}}\right\}-T_{d} \cos \theta \delta(x) \\
& +m_{b} g .
\end{aligned}
$$

For the convenience of research, the variables with subscripts $c$ and $b$ are defined as the variables of the cable and the deck beam, respectively. Variables $u$ and $v$ indicate the longitudinal and transverse vibration displacement, respectively, and they are the function of the position coordinate $x$ and time $t . X$ and $Y$ represent the longitudinal and transverse sag curves function, respectively; moreover, $Y=4 z_{0}[x / L-$ $\left.(x / L)^{2}\right]$. The meaning of other variables in formula (1) is as follows: $m, \omega, c, \xi, E, L, I, A, z_{0}, z$, and $g$ are the quality of unit length, natural frequency, damping coefficient, damping ratio, elastic modulus, length, moment of inertia, the cross section area, the transverse initial deflection at the midspan, the sag at the midspan, and gravitational acceleration, respectively. Units of each variable are taken from the international unit system. In addition, $T$ is the tangential tension of the stay cable, $T_{d}$ is the dynamic tension of cable in vibration process, $N$ is axial force of deck beam, $s$ is thr coordinate by the arc length, and $\delta(x)$ is Dirac function:

$$
\delta(x)= \begin{cases}\infty & x=0 \\ 0 & x \neq 0 .\end{cases}
$$

By using the static equilibrium relation of stay cable, formula (1) can be simplified as follows:

$$
\begin{aligned}
& m_{c} \frac{\partial^{2} u_{c}}{\partial t^{2}}+c_{c} \frac{\partial u_{c}}{\partial t} \\
& -\frac{\partial}{\partial s}\left\{T_{0} \frac{\partial u_{c}}{\partial s}+E_{c} A_{c} \varepsilon_{c}\left(\frac{d X_{c}}{d x_{c}}+\frac{\partial v_{c}}{\partial s}\right)\right\}=0 \\
& m_{c} \frac{\partial^{2} v_{c}}{\partial t^{2}}+c_{c} \frac{\partial v_{c}}{\partial t} \\
& -\frac{\partial}{\partial s}\left\{T_{0} \frac{\partial v_{c}}{\partial s}+E_{c} A_{c} \varepsilon_{c}\left(\frac{d Y_{c}}{d x_{c}}+\frac{\partial v_{c}}{\partial s}\right)\right\}=0 \\
& m_{b} \frac{\partial^{2} v_{b}}{\partial t^{2}}+c_{b} \frac{\partial v_{b}}{\partial t}+E_{b} I_{b} \frac{\partial^{4} v_{b}}{\partial x_{b}^{4}} \\
& -\frac{\partial}{\partial x_{b}}\left\{N \frac{\partial v_{b}}{\partial x_{b}}+E_{b} A_{b} \varepsilon_{b}\left(\frac{d Y_{b}}{d x_{c}}+\frac{\partial v_{b}}{\partial x_{b}}\right)\right\} \\
& +E_{c} A_{c} \varepsilon_{c} \cos \theta \delta(x)=0,
\end{aligned}
$$

where $T_{0}$ is the static tangential tension of stay cable in formula (3); moreover, $H_{0}$ is static axial tension and $\varepsilon_{c}$ and $\varepsilon_{b}$ are the axial strains of stay cable and beam, respectively. The axial strains can be expressed as follows:

$$
\begin{aligned}
& \varepsilon_{c}=\frac{\partial u_{c}}{\partial s}+\frac{d Y_{c}}{d x_{c}} \frac{\partial v_{c}}{\partial s}+\frac{1}{2}\left(\frac{\partial u_{c}}{\partial s}\right)^{2}+\frac{1}{2}\left(\frac{\partial v_{c}}{\partial s}\right)^{2} \\
& \varepsilon_{b}=\frac{\partial u_{b}}{\partial s}+\frac{d Y_{b}}{d x_{b}} \frac{\partial v_{b}}{\partial s}+\frac{1}{2}\left(\frac{\partial u_{b}}{\partial s}\right)^{2}+\frac{1}{2}\left(\frac{\partial v_{b}}{\partial s}\right)^{2}
\end{aligned}
$$


where $u_{b}$ represents the longitudinal displacement of beam in formula (4); the two-order small quantity of longitudinal elastic strain for the cable and beam can be neglected, because the longitudinal deformation of the cable and beam is much less than that of transverse deformation. It can be approximate to take $d s \approx d x$ for the stay cable with small sag, and formula (3) can be simplified as

$$
\begin{aligned}
& m_{c} \frac{\partial^{2} u_{c}}{\partial t^{2}}+c_{c} \frac{\partial u_{c}}{\partial t} \\
& -\frac{\partial}{\partial x_{c}}\left\{E_{c} A_{c}\left[\frac{\partial u_{c}}{\partial x_{c}}+\frac{d Y_{c}}{d x_{c}} \frac{\partial v_{c}}{\partial x_{c}}+\frac{1}{2}\left(\frac{\partial v_{c}}{\partial x_{c}}\right)^{2}\right] \frac{\partial v_{c}}{\partial x_{c}}\right\} \\
& =0 \\
& m_{c} \frac{\partial^{2} v_{c}}{\partial t^{2}}+c_{c} \frac{\partial v_{c}}{\partial t}-\frac{\partial}{\partial x_{c}}\left\{H_{0} \frac{\partial v_{c}}{\partial x_{c}}+E_{c} A_{c}\left(\frac{d Y_{c}}{d x_{c}}+\frac{\partial v_{c}}{\partial x_{c}}\right)\right. \\
& \left.\cdot\left[\frac{\partial u_{c}}{\partial x_{c}}+\frac{d Y_{c}}{d x_{c}} \frac{\partial v_{c}}{\partial x_{c}}+\frac{1}{2}\left(\frac{\partial v_{c}}{\partial x_{c}}\right)^{2}\right]\right\}=0 \\
& m_{b} \frac{\partial^{2} v_{b}}{\partial t^{2}}+c_{b} \frac{\partial v_{b}}{\partial t}+E_{b} I_{b} \frac{\partial^{4} v_{b}}{\partial x_{b}^{4}}-N \frac{\partial^{2} v_{b}}{\partial x_{b}^{2}}+E_{c} A_{c}\left[\frac{\partial u_{c}}{\partial x_{c}}\right. \\
& \left.+\frac{d Y_{c}}{d x_{c}} \frac{\partial v_{c}}{\partial x_{c}}+\frac{1}{2}\left(\frac{\partial v_{c}}{\partial x_{c}}\right)^{2}\right] \cos \theta \delta(x)=0 .
\end{aligned}
$$

In the case of low modal vibration for the cable, the coupling effect of transverse and longitudinal vibration for the cable is not considered. Meanwhile, the first equation of formula (5) can be simplified as

$$
e(t)=\frac{\partial u_{c}}{\partial x_{c}}+\frac{d Y_{c}}{d x_{c}} \frac{\partial v_{c}}{\partial x_{c}}+\frac{1}{2}\left(\frac{\partial v_{c}}{\partial x_{c}}\right)^{2} .
$$

The second equation of formula (5) can be simplified as

$$
\begin{aligned}
m_{c} \frac{\partial^{2} v_{c}}{\partial t^{2}}+c_{c} \frac{\partial v_{c}}{\partial t} & \\
& -\frac{\partial}{\partial x_{c}}\left\{H_{0} \frac{\partial v_{c}}{\partial x_{c}}+E_{c} A_{c}\left(\frac{d Y_{c}}{d x_{c}}+\frac{\partial v_{c}}{\partial x_{c}}\right) e(t)\right\}=0 .
\end{aligned}
$$

From the simplified assumptions, the geometric boundary condition of the stay cable is as follows:

$$
\begin{aligned}
u_{c}\left(L_{c}\right) & =0 ; \\
u_{c}(0)-u_{b}(0) \cos \theta & =0 .
\end{aligned}
$$

On both sides of formula (6) is integral on $\left[0, L_{c}\right]$ range for $x_{c}$ at the same time, and it is brought into formula (8), which can be obtained as follows:

$$
\begin{aligned}
e(t)= & \frac{v_{b}(0, t)}{L_{c}} \cos \theta \\
& +\frac{1}{L_{c}} \int_{0}^{L_{c}}\left[\frac{d Y_{c}}{d x_{c}} \frac{\partial v_{c}}{\partial x_{c}}+\frac{1}{2}\left(\frac{\partial v_{c}}{\partial x_{c}}\right)^{2}\right] d x_{c} .
\end{aligned}
$$

The axial dynamic force increment of the cable in the process of transverse vibration can be expressed as follows:

$$
\begin{aligned}
H_{d} & =\frac{E_{c} A_{c}}{L_{c e}}\left\{v_{b}(0, t) \cos \theta\right. \\
& \left.+\int_{0}^{L_{c}}\left[\frac{d Y_{c}}{d x_{c}} \frac{\partial v_{c}}{\partial x_{c}}+\frac{1}{2}\left(\frac{\partial v_{c}}{\partial x_{c}}\right)^{2}\right] d x_{c}\right\} \\
H_{\omega} & =\frac{E_{c} A_{c}}{L_{c e}} v_{b}(0, t) \cos \theta \\
H_{p} & =\frac{E_{c} A_{c}}{L_{c e}} \int_{0}^{L_{c}}\left[\frac{d Y_{c}}{d x_{c}} \frac{\partial v_{c}}{\partial x_{c}}+\frac{1}{2}\left(\frac{\partial v_{c}}{\partial x_{c}}\right)^{2}\right] d x_{c} .
\end{aligned}
$$

The axial force of bridge deck beam in vibration is as follows:

$$
N=\left(H+H_{d}\right) \sin \theta
$$

Equations (9) and (10) are simultaneous. The third equation of formula (5) is simplified; differential equations of motion for the cable-beam coupled vibration are as follows:

$$
\begin{aligned}
& m_{c} \ddot{v}_{c}+c_{c} \dot{v}_{c}-\left(H_{0}+H_{d}\right) \frac{\partial^{2} v_{c}}{\partial x_{c}^{2}}+H_{d} \frac{8 z_{0}}{L_{c}{ }^{2}}=0 \\
& m_{b} \ddot{v}_{b}+c_{b} \dot{v}_{b}+E_{b} I_{b} \frac{\partial^{4} v_{b}}{\partial x_{b}^{4}}-\left(H_{0}+H_{d}\right) \sin \theta \frac{\partial^{2} v_{b}}{\partial x_{b}^{2}} \\
& +H_{d} \cos \theta \delta(x)=0 .
\end{aligned}
$$

\subsection{Dimensionless Method of Parametric Resonance Equations} Considering Cable-Beam Coupling. The differential equation (12) of motion for cable-beam coupled vibrations is derived by using dimensionless method, and the following dimensionless quantities are defined [15-17]:

$$
\begin{aligned}
& \bar{t}=\omega_{c} t ; \\
& \bar{v}_{c}=\frac{v_{c}}{L_{c}} ; \\
& \bar{v}_{b}=\frac{v_{b}}{L_{b}} ; \\
& \bar{\omega}_{c}=\frac{\omega_{c e}}{\omega_{c}} ; \\
& \bar{\omega}_{b}=\frac{\omega_{b}}{\omega_{c}},
\end{aligned}
$$

where $\omega_{c}$ and $\omega_{c e}$ are the first natural frequencies of the cable without considering or considering the sag; $\omega_{b}$ is the first natural frequency of bridge deck beam; $\gamma$ is the sagspan ratio of cable; $k^{2}$ is the stiffness coefficient of cable; $L_{c}$ and $L_{b}$ are the span of cable and beam, respectively; $\lambda^{2}$ is Irvine parameter; $\beta_{b}$ indicates the ratio coefficient of first-order natural frequency for bridge deck beam; $\bar{t}$ denotes the dimensionless time; $\bar{v}_{c}$ and $\bar{v}_{b}$ are the transverse relative displacements of the cable and beam, respectively. $\bar{\omega}_{c}$ 
and $\bar{\omega}_{b}$ are the relative frequencies of the cable and beam, respectively.

The expressions of each parameter are

$$
\begin{aligned}
& \omega_{c}=\frac{\pi}{L_{c}} \sqrt{\frac{H_{0}}{m_{c}}} ; \\
& \omega_{c e}=\omega_{c}\left(1+\frac{(2 / \pi)^{4} \lambda^{2}}{2}\right)^{1 / 2} ; \\
& \omega_{b}=\beta_{b}^{2} \sqrt{\frac{E_{b} I_{b}}{m_{b} L_{b}^{4}}} ; \\
& \xi_{c}=\frac{c_{c}}{2 m_{c} \omega_{c}} ; \\
& \xi_{b}=\frac{c_{b}}{2 m_{b} \omega_{b}} ; \\
& \gamma=\frac{z_{0}}{L_{c}} ; \\
& k^{2}=\frac{E_{c} A_{c}}{H_{0}} ; \\
& \lambda_{b}^{4}=\frac{64 k^{2} \gamma^{2}}{1+8 \gamma^{2}} ; \\
& E_{b} I_{b}
\end{aligned}
$$

Equation (12) is derived by using the dimensionless quantity of the above definition.

$$
\begin{aligned}
& \frac{\partial^{2} \bar{v}_{c}}{\partial \bar{t}^{2}}+2 \xi_{c} \bar{\omega}_{c} \frac{\partial \bar{v}_{c}}{\partial \bar{t}}-\frac{1}{\pi^{2}}\left(1+\bar{H}_{\omega}+\bar{H}_{p}\right) \frac{\partial^{2} \bar{v}_{c}}{\partial \bar{x}_{c}^{2}} \\
& +\left(\bar{H}_{\omega}+\bar{H}_{p}\right) \frac{8 \gamma}{\pi^{2}}=0 \\
& \frac{\partial^{2} \bar{v}_{b}}{\partial \bar{t}^{2}}+2 \xi_{b} \bar{\omega}_{b} \frac{\partial \bar{v}_{c}}{\partial \bar{t}}+\frac{1}{\beta_{b}^{4}} \bar{\omega}_{b}^{2} \frac{\partial^{4} \bar{v}_{b}}{\partial \bar{x}_{b}^{4}} \\
& \quad-\frac{1}{\pi^{2}}\left(1+\bar{H}_{\omega}+\bar{H}_{p}\right)\left(\frac{m_{c}}{m_{b}}\right)\left(\frac{L_{c}}{L_{b}}\right)^{2} \sin \theta \frac{\partial^{2} \bar{v}_{b}}{\partial \bar{x}_{b}^{2}} \\
& +\frac{1}{\pi^{2}}\left(\bar{H}_{\omega}+\bar{H}_{p}\right)\left(\frac{m_{c}}{m_{b}}\right)\left(\frac{L_{c}^{2}}{L_{b}}\right) \cos \theta \delta(x)=0 \\
& \bar{H}_{\omega}=\frac{k^{2}}{1+8 \gamma^{2}} \bar{v}_{b}(0, \bar{t}) \cos \theta \\
& \bar{H}_{p}=\frac{\lambda^{2}}{64}\left\{8 \int_{0}^{1} \frac{1}{\gamma} \bar{v}_{c} d \bar{x}_{c}+\frac{1}{2} \int_{0}^{1}\left(\frac{1}{\gamma} \frac{\partial \bar{v}_{c}}{\partial \bar{x}_{c}}\right)^{2} d \bar{x}_{c}\right\} .
\end{aligned}
$$

When considering that the first-order mode is only the main vibration, and constraint on the boundary condition of formula (8) is satisfied, the dimensionless displacement function of the cable is as follows:

$$
\bar{v}_{c}\left(\bar{x}_{c}, \bar{t}\right)=\bar{v}_{b}(0, \bar{t}) \sin \theta\left(1-\bar{x}_{c}\right)+\sin \left(\pi \bar{x}_{c}\right) q_{c}(\bar{t}) .
$$

Moreover, the geometric boundary condition of the fixed end for the bridge deck beam is as follows:

$$
\begin{gathered}
v_{b}\left(L_{b}\right)=0 ; \\
\dot{v}_{b}\left(L_{b}\right)=0 ; \\
\ddot{v}_{b}(0)=0 .
\end{gathered}
$$

The physical boundary condition of the connection between cable and bridge deck beam is as follows:

$$
E_{b} I_{b} v_{b}^{\prime \prime \prime}(0)=-k_{c} v_{b}(0),
$$

while the dimensionless modal function of in-plane vibration for the deck beam can be expressed as follows:

$$
\begin{aligned}
\bar{\psi}_{b}\left(\bar{x}_{b}\right)= & A_{1} \sin \left(\beta_{b} \bar{x}_{b}\right)+A_{2} \cos \left(\beta_{b} \bar{x}_{b}\right) \\
& +A_{3} \sinh \left(\beta_{b} \bar{x}_{b}\right)+A_{4} \cosh \left(\beta_{b} \bar{x}_{b}\right),
\end{aligned}
$$

where $A_{1}, A_{2}, A_{3}$, and $A_{4}$ are constant coefficients; they are determined by formulas (17) and (18). Moreover, $\beta_{b}$ is determined by the following characteristic equation:

$$
\frac{k_{c}}{E_{b} I_{b}}=\beta_{b}^{3} \frac{1+\cos \beta_{b} L_{b} \cosh \beta_{b} L_{b}}{\cos \beta_{b} L_{b} \sinh \beta_{b} L_{b}-\sin \beta_{b} L_{b} \cosh \beta_{b} L_{b}} .
$$

And the dimensionless displacement function of the deck beam can be expressed as follows:

$$
\bar{v}_{b}\left(\bar{x}_{b}, \bar{t}\right)=\bar{\psi}_{b}\left(\bar{x}_{b}\right) \bar{q}_{b}(\bar{t}) .
$$

Formulas (16) and (21) are brought into formula (12) by the Galerkin method, on both sides multiplied by $\sin \left(\pi \bar{x}_{c}\right)$ and $\bar{\psi}_{b}$ at the same time, and integral on $[0,1]$; it can be obtained by using the mode orthogonality principle:

$$
\begin{aligned}
\ddot{q}_{c} & +2 \xi_{c} \bar{\omega}_{c} \dot{q}_{c}+\bar{\omega}_{c}^{2} q_{c}+\alpha_{11} q_{c} q_{b}+\alpha_{12} q_{c} q_{b}^{2}+\alpha_{2} q_{c}^{2} \\
& +\alpha_{3} q_{c}^{3}+\alpha_{4} q_{b}+\alpha_{5} q_{b}^{2}+\gamma_{1} \dot{q}_{b}+\gamma_{2} \ddot{q}_{b}=0 \\
\ddot{q}_{b} & +2 \xi_{b} \bar{\omega}_{b} \dot{q}_{b}+\bar{\omega}_{b}^{2} q_{b}+\beta_{1} q_{b}+\beta_{11} q_{c} q_{b}+\beta_{12} q_{c}^{2} q_{b} \\
& +\beta_{2} q_{b}^{2}+\beta_{3} q_{b}^{3}+\beta_{4} q_{c}+\beta_{5} q_{c}^{2}=0,
\end{aligned}
$$

where $\alpha_{11}, \alpha_{12}, \alpha_{2}, \alpha_{3}, \alpha_{4}, \alpha_{5}, \gamma_{1}, \gamma_{2}, \zeta_{1}, \zeta_{2}, \mu_{1}, \mu_{2}, \beta_{1}, \beta_{11}, \beta_{12}$, $\beta_{2}, \beta_{3}, \beta_{4}$, and $\beta_{5}$ are parameters.

\section{Discussion on the Equations with Dimensionless Parametric Resonance}

3.1. Approximate Solution of the Method of Multiple Scales. The approximate solution of formula (22) is solved by the method of multiple scales, setting the form of solution as follows:

$$
\begin{aligned}
q_{c}= & \varepsilon q_{c 1}\left(T_{0}, T_{1}, T_{2}\right)+\varepsilon^{2} q_{c 2}\left(T_{0}, T_{1}, T_{2}\right) \\
& +\varepsilon^{3} q_{c 3}\left(T_{0}, T_{1}, T_{2}\right) \\
q_{b}= & \varepsilon q_{b 1}\left(T_{0}, T_{1}, T_{2}\right)+\varepsilon^{2} q_{b 2}\left(T_{0}, T_{1}, T_{2}\right) \\
& +\varepsilon^{3} q_{b 3}\left(T_{0}, T_{1}, T_{2}\right) .
\end{aligned}
$$


Before the multiscale method is adopted to solve the equation, the parameters are as follows:

$$
\begin{aligned}
& \xi_{c}=\varepsilon^{2} \mu_{c} ; \\
& \xi_{b}=\varepsilon^{2} \mu_{b} ; \\
& \alpha_{4}=\varepsilon \eta_{1} ; \\
& \gamma_{1}=\varepsilon^{2} \eta_{2} ; \\
& \gamma_{2}=\varepsilon^{2} \eta_{3} ; \\
& \beta_{1}=\varepsilon \eta_{4} ; \\
& \beta_{4}=\varepsilon \eta_{5} .
\end{aligned}
$$

Formula (23) is brought into formula (22), and the same power coefficient of $\varepsilon$ is sorted out, which can be obtained as follows:

$\varepsilon$ order is

$$
\begin{aligned}
& D_{0}^{2} q_{c 1}+\bar{\omega}_{c}^{2} q_{c 1}=0 \\
& D_{0}^{2} q_{b 1}+\bar{\omega}_{b}^{2} q_{b 1}=0 .
\end{aligned}
$$

$\varepsilon^{2}$ order is

$$
\begin{aligned}
D_{0}^{2} q_{c 2}+\bar{\omega}_{c}^{2} q_{c 2}= & -2 D_{0} D_{1} q_{c 1}-\alpha_{11} q_{c 1} q_{b 1}-\alpha_{2} q_{c 1}^{2} \\
& -\eta_{1} q_{b 1}-\alpha_{5} q_{b 1}^{2} \\
D_{0}^{2} q_{b 2}+\bar{\omega}_{b}^{2} q_{b 2}= & -2 D_{0} D_{1} q_{b 1}-\eta_{4} q_{b 1}-\beta_{11} q_{c 1} q_{b 1} \\
& -\beta_{2} q_{b 1}^{2}-\eta_{5} q_{c 1}-\beta_{5} q_{c 1}^{2} .
\end{aligned}
$$

$\varepsilon^{3}$ order is

$$
\begin{aligned}
D_{0}^{2} q_{c 3}+\bar{\omega}_{c}^{2} q_{c 3}= & -2 D_{0} D_{1} q_{c 2}-\left(D_{1}^{2}+2 D_{0} D_{2}\right) q_{c 1} \\
& -2 \mu_{c} \bar{\omega}_{c} D_{0} q_{c 1} \\
& -\alpha_{11}\left(q_{c 1} q_{b 2}+q_{c 2} q_{b 1}\right) \\
& -\alpha_{12} q_{c 1} q_{b 1}^{2}-2 \alpha_{2} q_{c 1} q_{c 2}-\alpha_{3} q_{c 1}^{3} \\
& -\eta_{1} q_{b 2}-2 \alpha_{5} q_{b 1} q_{b 2}-\eta_{2} D_{0} q_{b 1} \\
& -\eta_{3} D_{0}^{2} q_{b 1} \\
D_{0}^{2} q_{b 3}+\bar{\omega}_{b}^{2} q_{b 3}= & -2 D_{0} D_{1} q_{b 2}-\left(D_{1}^{2}+2 D_{0} D_{2}\right) q_{b 1} \\
& -2 \mu_{b} \bar{\omega}_{b} D_{0} q_{b 1} \\
& -\beta_{11}\left(q_{c 1} q_{b 2}+q_{c 2} q_{b 1}\right)-\eta_{4} q_{b 2} \\
& -\beta_{12} q_{c 1}^{2} q_{b 1}-2 \beta_{2} q_{b 1} q_{b 2}-\beta_{3} q_{b 1}^{3} \\
& -\eta_{5} q_{c 2}-2 \beta_{5} q_{c 1} q_{c 2} .
\end{aligned}
$$

From formula (27), the first solution of the equation can be expressed as

$$
\begin{aligned}
& q_{c 1}=Q_{1}\left(T_{1}, T_{2}\right) e^{i \bar{\omega}_{c} T_{0}}+c c \\
& q_{b 1}=B_{1}\left(T_{1}, T_{2}\right) e^{i \bar{\omega}_{b} T_{0}}+c c,
\end{aligned}
$$

where $c c$ represents the front of the conjugate.

Formula (28) is brought into the second equations of formula (27) and can be obtained as follows:

$$
\begin{aligned}
D_{0}^{2} q_{c 2}+\bar{\omega}_{c}^{2} q_{c 2}= & -i 2 \bar{\omega}_{c} \frac{\partial Q_{1}}{\partial T_{1}} e^{i \bar{\omega}_{c} T_{0}}-\alpha_{2} Q_{1}^{2} e^{i 2 \bar{\omega}_{c} T_{0}} \\
& -\eta_{1} B_{1} e^{i \bar{\omega}_{b} T_{0}}-\alpha_{5} B_{1}^{2} e^{i 2 \bar{\omega}_{b} T_{0}} \\
& -\alpha_{11} Q_{1} B_{1} e^{i\left(\bar{\omega}_{c}+\bar{\omega}_{b}\right) T_{0}} \\
& -\alpha_{11} \bar{Q}_{1} B_{1} e^{i\left(\bar{\omega}_{b}-\bar{\omega}_{c}\right) T_{0}} \\
& -\left(\alpha_{2} Q_{1} \bar{Q}_{1}+\alpha_{5} B_{1} \bar{B}_{1}\right)+c c \\
D_{0}^{2} q_{b 2}+\bar{\omega}_{b}^{2} q_{b 2}= & -i 2 \bar{\omega}_{b} \frac{\partial B_{1}}{\partial T_{1}} e^{i \bar{\omega}_{b} T_{0}}-\eta_{4} B_{1} e^{i \bar{\omega}_{b} T_{0}} \\
& -\beta_{2} B_{1}^{2} e^{i 2 \bar{\omega}_{b} T_{0}}-\eta_{5} Q_{1} e^{i \bar{\omega}_{c} T_{0}} \\
& -\beta_{5} Q_{1}^{2} e^{i 2 \bar{\omega}_{c} T_{0}} \\
& -\beta_{11} Q_{1} B_{1} e^{i\left(\bar{\omega}_{c}+\bar{\omega}_{b}\right) T_{0}} \\
& -\beta_{11} \bar{Q}_{1} B_{1} e^{i\left(\bar{\omega}_{b}-\bar{\omega}_{c}\right) T_{0}} \\
& -\left(\beta_{5} Q_{1} \bar{Q}_{1}+\beta_{2} B_{1} \bar{B}_{1}\right)+c c .
\end{aligned}
$$

It can be obtained by eliminating the periodic term of formula (29):

$$
\begin{array}{r}
\frac{\partial Q_{1}}{\partial T_{1}}=0 \\
i 2 \bar{\omega}_{b} \frac{\partial B_{1}}{\partial T_{1}}+\eta_{4} B_{1}=0 .
\end{array}
$$

By using the method that the equation contains a nonperiodic term of multiple scales, when the internal resonance is not considered, the solution of formula (29) is as follows:

$$
\begin{aligned}
q_{c 2}= & \frac{\alpha_{2} Q_{1}^{2}}{3 \bar{\omega}_{c}^{2}} e^{i 2 \bar{\omega}_{c} T_{0}}+\frac{\eta_{1} B_{1}}{\left(\bar{\omega}_{b}^{2}-\bar{\omega}_{c}^{2}\right)} e^{i \bar{\omega}_{b} T_{0}} \\
& +\frac{\alpha_{5} B_{1}^{2}}{\left(4 \bar{\omega}_{b}^{2}-\bar{\omega}_{c}^{2}\right)} e^{i 2 \bar{\omega}_{b} T_{0}} \\
& +\frac{\alpha_{11} Q_{1} B_{1}}{\left(\bar{\omega}_{b}^{2}+2 \bar{\omega}_{c} \bar{\omega}_{b}\right)} e^{i\left(\bar{\omega}_{c}+\bar{\omega}_{b}\right) T_{0}} \\
& +\frac{\alpha_{11} \bar{Q}_{1} B_{1}}{\left(\bar{\omega}_{b}^{2}-2 \bar{\omega}_{c} \bar{\omega}_{b}\right)} e^{i\left(\bar{\omega}_{b}-\bar{\omega}_{c}\right) T_{0}}
\end{aligned}
$$


TABLE 1: Parameter of cables and bridge deck beam.

\begin{tabular}{|c|c|c|c|}
\hline Cable length (m) & Deck beam length (m) & Cable quality $\left(\mathrm{kg} \cdot \mathrm{m}^{-1}\right)$ & $\begin{array}{l}\text { Bridge deck beam } \\
\text { quality }\left(\mathrm{kg} \cdot \mathrm{m}^{-1}\right)\end{array}$ \\
\hline 178 & 30 & 120.4 & $1.6 \times 10^{6}$ \\
\hline Elastic modulus of cable $(\mathrm{GPa})$ & Elastic modulus of bridge deck (GPa) & Cable section area $\left(\mathrm{cm}^{2}\right)$ & Angle of cable $\left(^{\circ}\right)$ \\
\hline 190 & 35 & 141.24 & 62.1 \\
\hline Natural frequency of cable $\omega_{c}(\mathrm{~Hz})$ & Cable damping ratio $\xi_{c}$ & Damping ratio of bridge deck $\xi_{b}$ & Time step $\Delta \bar{t}$ \\
\hline 0.768 & 0.001 & 0.01 & 0.01 \\
\hline
\end{tabular}

$$
\begin{aligned}
& -\frac{\alpha_{2} Q_{1} \bar{Q}_{1}+\alpha_{5} B_{1} \bar{B}_{1}}{\bar{\omega}_{c}^{2}}+c c \\
q_{b 2}= & \frac{\beta_{2} B_{1}^{2}}{3 \bar{\omega}_{b}^{2}} e^{i 2 \bar{\omega}_{b} T_{0}}-\frac{\eta_{5} Q_{1}}{\left(\bar{\omega}_{b}^{2}-\bar{\omega}_{c}^{2}\right)} e^{i \bar{\omega}_{c} T_{0}} \\
& -\frac{\beta_{5} Q_{1}^{2}}{\left(\bar{\omega}_{b}^{2}-4 \bar{\omega}_{c}^{2}\right)} e^{i 2 \bar{\omega}_{c} T_{0}} \\
& +\frac{\beta_{11} Q_{1} B_{1}}{\left(\bar{\omega}_{c}^{2}+2 \bar{\omega}_{c} \bar{\omega}_{b}\right)} e^{i\left(\bar{\omega}_{c}+\bar{\omega}_{b}\right) T_{0}} \\
& +\frac{\beta_{11} \bar{Q}_{1} B_{1}}{\left(\bar{\omega}_{c}^{2}-2 \bar{\omega}_{c} \bar{\omega}_{b}\right)} e^{i\left(\bar{\omega}_{b}-\bar{\omega}_{c}\right) T_{0}} \\
& -\frac{\left(\beta_{5} Q_{1} \bar{Q}_{1}+\beta_{2} B_{1} \bar{B}_{1}\right)}{\bar{\omega}_{b}^{2}}+c c
\end{aligned}
$$

\subsection{Discussion on Resonance Characteristics of Parametric} Excitation. From formula (31), $\left(\eta_{1} B_{1} /\left(\bar{\omega}_{b}^{2}-\bar{\omega}_{c}^{2}\right)\right) e^{i \bar{\omega}_{b} T_{0}}$ items contained in the expression of the displacement modal component $q_{c 2}$ of the cable will make the first equation show the nonsingular solution when the frequency ratio between the bridge and the cable is satisfied with $\bar{\omega}_{b}: \bar{\omega}_{c}=1: 1$. It can be seen from the second equations that $-\left(\eta_{5} Q_{1} /\left(\bar{\omega}_{b}^{2}-\right.\right.$ $\left.\left.\bar{\omega}_{c}^{2}\right)\right) e^{i \bar{\omega}_{c} T_{0}}$ items contained in the expression of the displacement modal components $q_{b 2}$ of the bridge deck beam will make the second equation show the nonsingular solution, and this moment the cable-beam coupling system will present the $1: 1$ principal parametric resonance phenomenon with displacement modal component $q_{c 2}$ and $q_{b 2}$ as the main form.

Similarly, when the frequency ratio between the bridge deck beam and the cable is satisfied with the $\bar{\omega}_{b}: \bar{\omega}_{c}=$ $2: 1$ and $\bar{\omega}_{b}: \bar{\omega}_{c}=1: 2,\left(\alpha_{11} \bar{Q}_{1} B_{1} /\left(\bar{\omega}_{b}^{2}-2 \bar{\omega}_{c} \bar{\omega}_{b}\right)\right) e^{i\left(\bar{\omega}_{b}-\bar{\omega}_{c}\right) T_{0}}$ or $\left(\alpha_{5} B_{1}^{2} /\left(4 \bar{\omega}_{b}^{2}-\bar{\omega}_{c}^{2}\right)\right) e^{i 2 \bar{\omega}_{b} T_{0}}$ items of the first equation and $-\left(\beta_{5} Q_{1}^{2} /\left(\bar{\omega}_{b}^{2}-4 \bar{\omega}_{c}^{2}\right)\right) e^{i 2 \bar{\omega}_{c} T_{0}}$ or $\left(\beta_{11} \bar{Q}_{1} B_{1} /\left(\bar{\omega}_{c}^{2} \quad-\right.\right.$ $\left.\left.2 \bar{\omega}_{c} \bar{\omega}_{b}\right)\right) e^{i\left(\bar{\omega}_{b}-\bar{\omega}_{c}\right) T_{0}}$ items of the second equation of formula (31) led to the equations showing nonsingular solution.

At this moment, the cable-beam coupling system will present the large vibration phenomenon of the $2: 1$ principal parametrical resonance and $1: 2$ superharmonic resonance with displacement modal component $q_{c 2}$ and $q_{b 2}$ as the main form.

\section{Analysis on Numerical Example}

4.1. Basic Parameters. The cable in practical engineering of a cable-stayed bridge can be taken as the study object, in order to further verify the characteristics of $1: 1$ main parametrical resonance for cable-beam coupled system. The geometric parameters and material properties of the stay cable are shown in Table 1.

\subsection{The 1:1 Principal Parametrical Resonance Response Char- acteristics of Cable-Beam Coupled System}

4.2.1. When the Working Conditions $q_{c}(0)=5.618 \times 10^{-6}$ and $q_{b}(0)=3.33 \times 10^{-4}$. Under this working condition, the actual initial displacements of cable midspan and deck beam end are about $0.001 \mathrm{~m}$ and $0.01 \mathrm{~m}$. The dimensionless displacement response time history and amplitude-frequency characteristic curve of coupled system are shown in Figures 2 and 3.

From cable's dimensionless displacement response time history and spectrum curve in Figure 2, stay cable presents large beat vibration near the equilibrium position at its natural frequency $\omega_{c}$. Due to the small damping of the cable and the slow decay of parametrical resonance displacement response, the sharp vibration has been continued. From the dimensionless displacement response time history and amplitude-frequency characteristic curve of the deck beam end in Figure 3, beam presents the obvious beat vibration near the equilibrium position at initial displacement, the coupling effect is obvious, and the amplitude-frequency characteristic curve of the deck beam end is in single peak value, which says that it does harmonic vibration at its natural frequency.

We could find that, from the dimensionless displacement response amplitude of cable-beam coupled system, due to deck beam's large quality and stiffness, its energy during the vibration is larger than the energy during cable's parametrical resonance process when its excitation amplitude of vertical initial displacement is small; at this moment, the constraints of the cable on the deck beam movement are very small, while the effect of the deck beam on the cable movement is large, so cable presents large beat vibration, and deck beam presents small beat vibration at given initial conditions.

4.2.2. When the Working Conditions $q_{c}(0)=5.618 \times 10^{-6}$ and $q_{b}(0)=1.66 \times 10^{-3}$. Under this working condition, the actual initial displacements of cable midspan and deck beam end are about $0.001 \mathrm{~m}$ and $0.05 \mathrm{~m}$. The dimensionless 

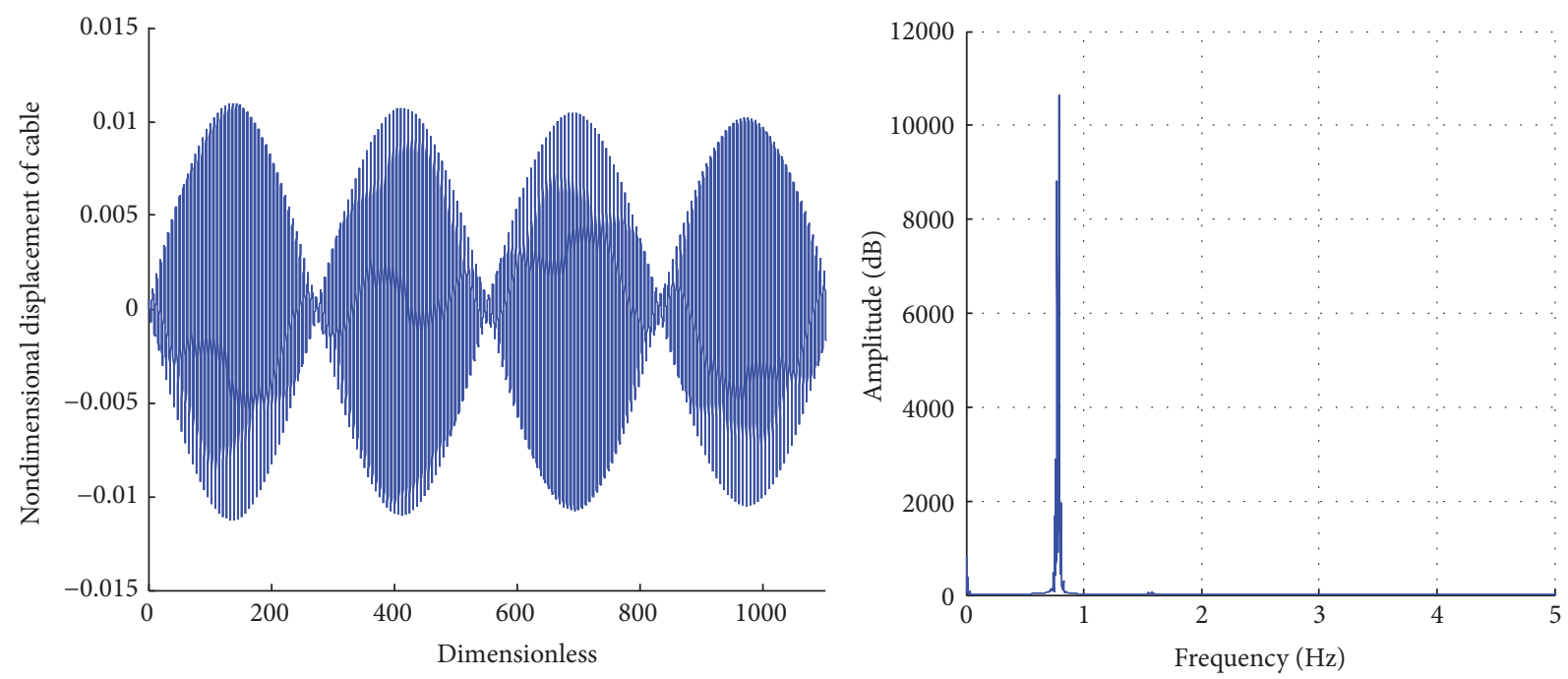

Figure 2: Dimensionless displacement response time history and amplitude-frequency characteristic curve of the cable midspan.
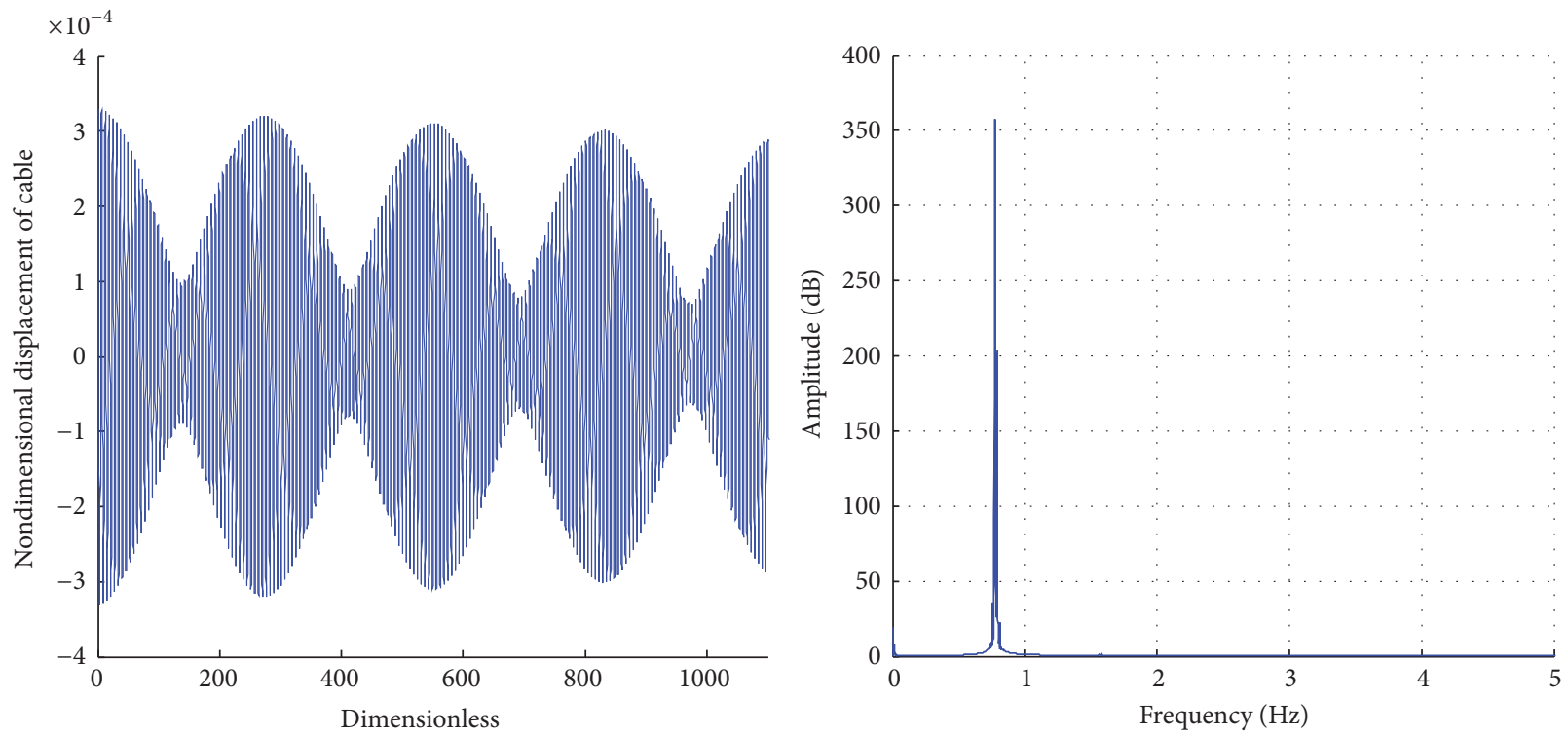

FIGURE 3: Dimensionless displacement response time history and amplitude-frequency characteristic curve of the deck beam end.

displacement response time history and amplitude-frequency characteristic curve of coupling system are shown in Figures 4 and 5 .

From Figures 5 and 6, compared with working condition $q_{b}(0)=3.3 \times 10^{-4}$, parametrical resonance's coupling effect of cable-beam composite structure is much more obvious under this working condition. Both cable and deck beam present obvious beat vibration phenomenon, but cable's beat vibration has greater displacement response; the maximal displacement amplitude is increased from $1.18 \%$ of the cable length to $2.42 \%$, which says that cable midspan's parametrical resonance displacement amplitude is also increased as the excitation amplitude of vertical initial displacement of deck beam end increased; this is fully consistent with the principle of conservation of energy.
4.2.3. When the Working Conditions $q_{c}(0)=5.618 \times 10^{-6}$ and $q_{b}(0)=3.3 \times 10^{-8}$. Under this working condition, the actual initial displacements of cable midspan and deck beam end are about $0.001 \mathrm{~m}$ and $10^{-6} \mathrm{~m}$. The dimensionless displacement response time history and amplitude-frequency characteristic curve of coupling system are shown in Figures 6 and 7.

From Figures 6 and 7, under the condition of beam's small initial displacement, both cable and beam present obvious beat vibration phenomenon; the coupling effect is obvious, but at this point the cable's displacement amplitude is small and there is no significant vibration, which is only a slight stable "beat" vibration in the vicinity of the equilibrium position. Analysis of the reason is as follows: from the multiscale differential equations (29) of stay cable 

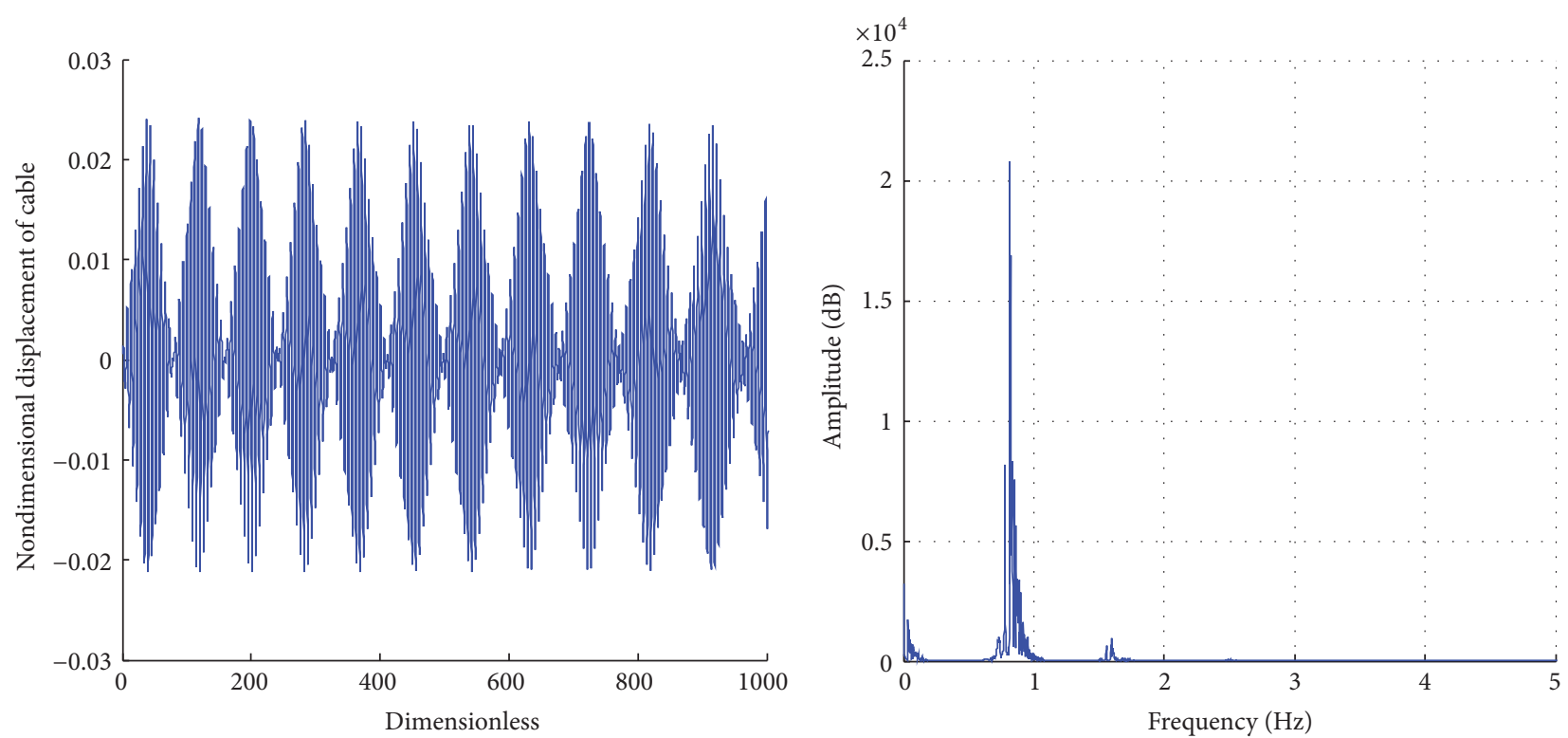

FIGURE 4: Dimensionless displacement response time history and amplitude-frequency characteristic curve of the cable midspan.
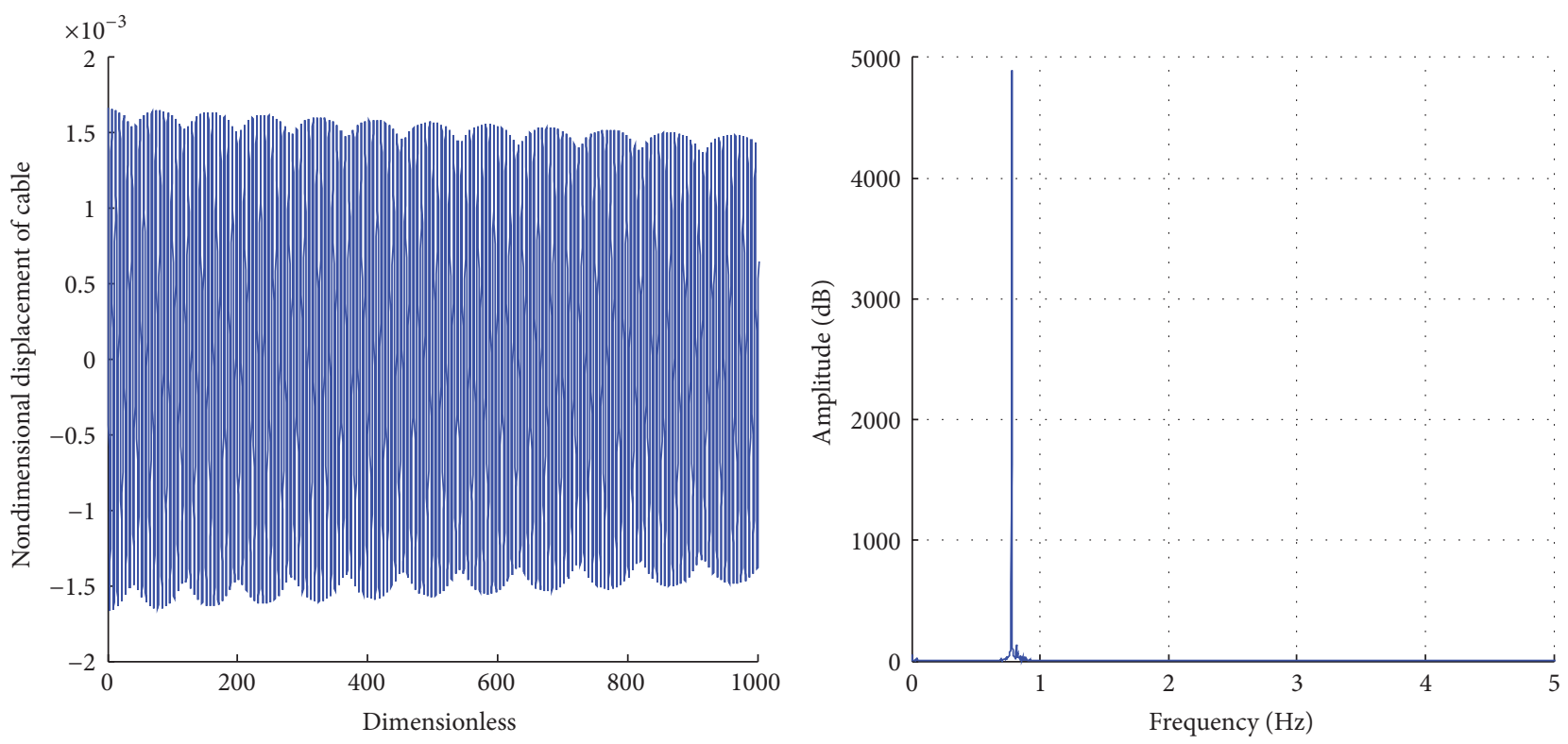

FIGURE 5: Dimensionless displacement response time history and amplitude-frequency characteristic curve of the deck beam end.

and beam's displacement components $q_{c 2}$ and $q_{b 2}$, resonance terms of both parametrical resonance and primary resonance $-\alpha_{11} \bar{Q}_{1} B_{1} e^{i\left(\bar{\omega}_{b}-\bar{\omega}_{c}\right) T_{0}}$ and $-\eta_{1} B_{1} e^{i \bar{\omega}_{b} T_{0}}$ are related to the coefficient $\alpha_{11}$ affected by deck beam's excitation amplitude. By derivation, we conclude that the coefficient $32 \gamma / \pi^{3}$ which is the difference between coefficients $\eta_{1}$ and $\alpha_{11}$ is much affected by sag. So the main parametrical resonance will be not significant vibration under beam's small initial condition.

\section{Conclusions}

(1) Based on the establishment of the model of the cablebeam coupling dimensionless parametrical resonance, the main parametrical resonance characteristics of cable-beam coupled system are discussed emphatically in terms of the frequency ratio relation of the deck beam and cable meets $\bar{\omega}_{b}: \bar{\omega}_{c}=1: 1$. Then, the possibility of the occurrence of large main parametrical resonance of cable is proved theoretically. 

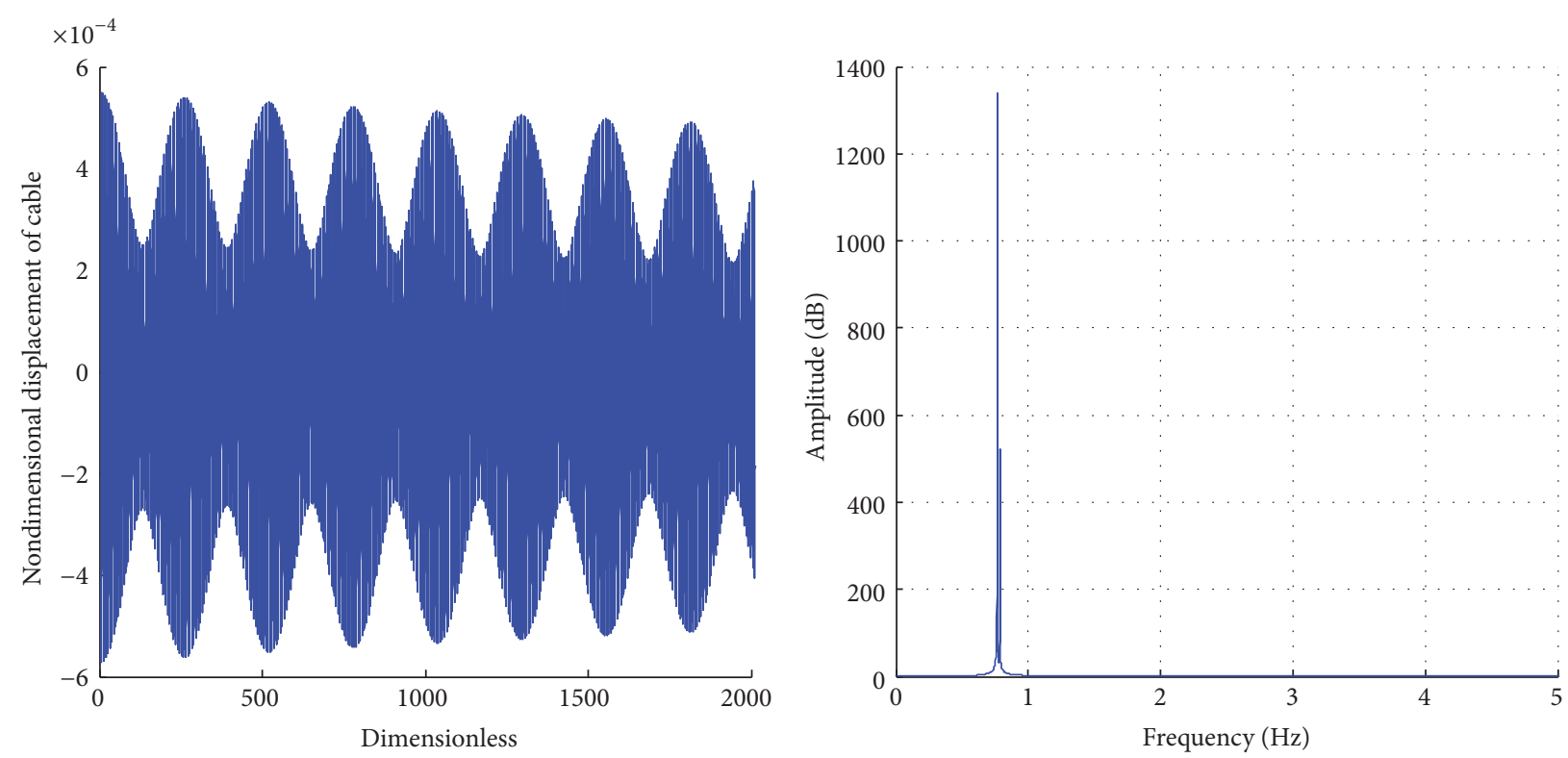

Figure 6: Dimensionless displacement response time history and amplitude-frequency characteristic curve of the cable midspan.
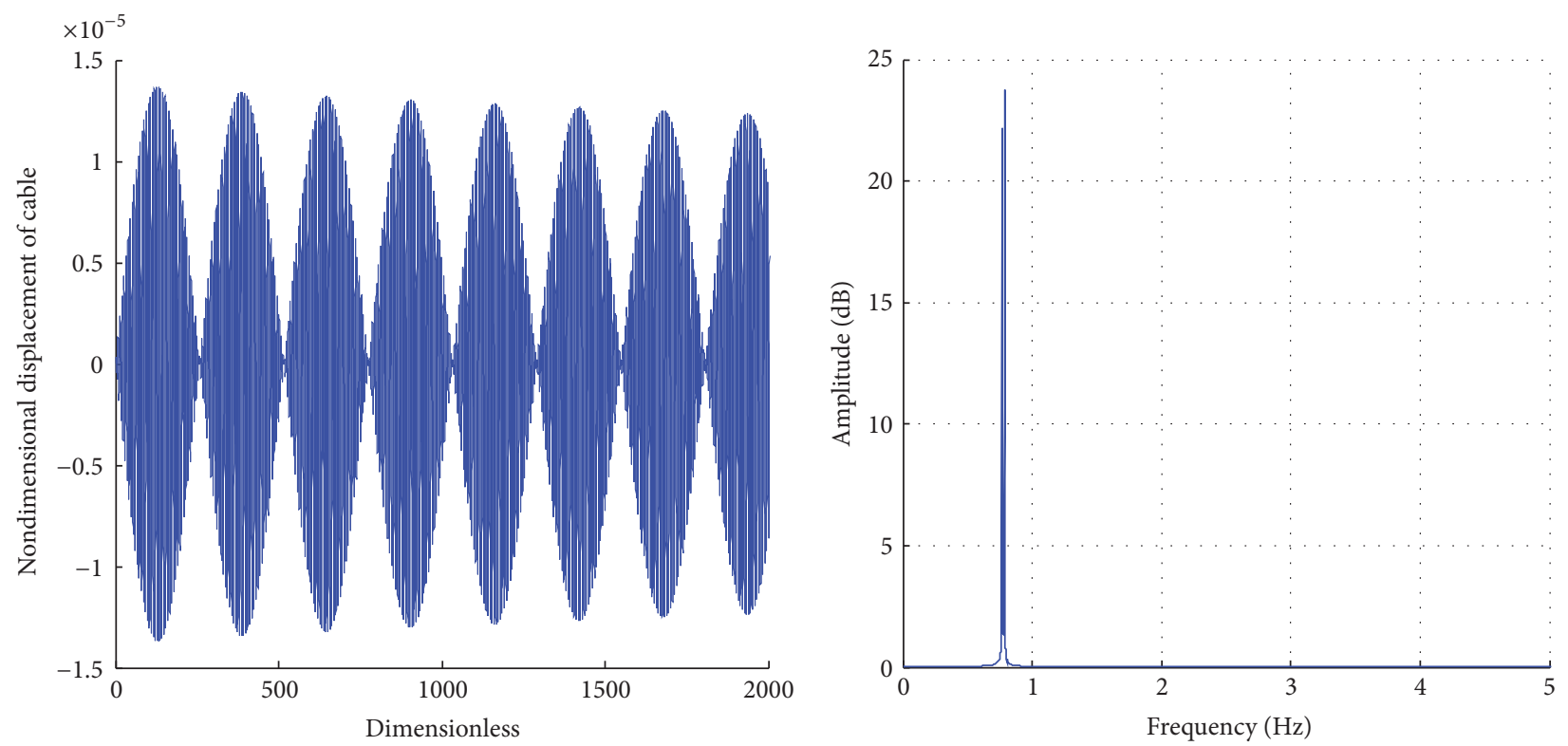

Figure 7: Dimensionless displacement response time history and amplitude-frequency characteristic curve of the deck beam end.

(2) The nonlinear main parametrical resonance response of cable is irrelevant to its initial displacement, which is increased with the bigger initial displacement of the deck beam end; also, the cable midspan displacement is increased obviously and presents obvious beat vibration phenomenon.

(3) When the vertical initial displacement of the deck beam end is $10^{-6} \mathrm{~m}$ or even smaller, both the cable and beam present obvious beat vibration phenomenon, but, at this time, the cable displacement amplitude is small and there is no significant vibration, only making a slight stable "beat" vibration in the vicinity of the equilibrium position, which is different from the situation of $2: 1$ parametrical resonance.

\section{Competing Interests}

The authors declare that they have no competing interests.

\section{Acknowledgments}

This research was financed by National Natural Science Foundation of China (51468001), the Science and Technology Support Program of Jiangxi Province (20122BAB216004, 20133BBE50032, and 20141BBE50022), and Young Scientists (Jing Gang star) Training Object Program of Jiangxi Province (20133BCB23021). This research was supported by Basic Science Research Program through the National Research 
Foundation of Korea (NRF) funded by the Ministry of Science, ICT \& Future Planning (no. 2015R1A5A1037548).

\section{References}

[1] H. Max Irvine, Cable Structures, MIT Press, Cambridge, Mass, USA, 1981.

[2] T.-Z. Liu and L.-Q. Chen, Nonlinear Vibrations, Higher Education Press, Beijing, China, 2001.

[3] Y.-S. Chen, Nonlinear Vibrations, Higher Education Press, Beijing, China, 2002.

[4] Z. Kang and W.-X. Zhong, "Numerical study on parametric resonance of cable stayed bridge," China Civil Engineering Journal, vol. 31, no. 4, pp. 14-22, 1998.

[5] S.-S. Chen and B.-N. Sun, "Numerical study on nonlinear parametric vibration of coupled cables and bridge decks," China Civil Engineering Journal, vol. 36, no. 4, pp. 70-75, 2003.

[6] Z.-X. Li, H.-Q. Chen, and Y.-T. Li, "Finite element analysis and semi-active control of parametric vibrations of cable-stayed bridges," Engineering Mechanics, vol. 21, no. 1, pp. 131-135, 2004.

[7] W.-L. Chen and H. Li, "Passive control for parametric vibration of cables using viscous fluid dampers," Earthquake Engineering and Engineering Vibration, vol. 27, no. 2, pp. 137-144, 2006.

[8] F. Li, O. Yang, S. Tian, and L. Zhang, "Nonlinear dynamic response analysis of cable-bridge coupling based on multiple scale method," Journal of Shenyang Jianzhu University (Natural Science), vol. 29, no. 5, pp. 816-822, 2013.

[9] F.-C. Li, O. Yang, S.-Z. Tian et al., "Nonlinear parametric vibration of cable with consideration of first two orders of modal combination," Journal of Disaster Prevention and Mitigation Engineer, vol. 35, no. 2, pp. 249-255, 2015.

[10] Y.-Y. Zhao, X.-Z. Yang, W.-C. Liu, and L.-H. Wang, "Non-linear response of cables in cable-stayed beam structure," Gongcheng Lixue/Engineering Mechanics, vol. 23, no. 11, pp. 153-158, 2006.

[11] H.-J. Kang, Y.-Y. Zhao, and L.-Z. Jiang, "In-plane nonlinear vibration of super long stay cables under parametric and forced excitations," Journal of Central South University (Science and Technology), vol. 42, no. 8, pp. 2439-2445, 2011.

[12] Q.-X. Wu, W.-P. Wang, and B.-C. Chen, "Finite element analysis for nonlinear vibration of cable-beam structure," Gongcheng Lixue/Engineering Mechanics, vol. 30, no. 3, pp. 347-382, 2013.

[13] Y.-Y. Zhao, L.-Z. Jiang, L.-H. Wang et al., "The dynamical modelling theory and internal resonance of cable-beam composite structure," China Civil Engineering Journal, vol. 37, no. 3, pp. 69104, 2004.

[14] H.-B. Zhou, The experimental investigation on nonlinear dynamics of cable-beam structure [Ph.D. dissertation], Hunan University, Changsha, China, 2007.

[15] F.-C. Li, Parametric vibration and cable force identification of staying cable of large-span bridges [Ph.D. thesis], Harbin Institute of Technology, Harbin, China, 2009.

[16] A. Y. Koverga and E. P. Kubyshkin, "Features of the behavior of solutions to a nonlinear dynamical system in the case of two frequency parametric resonance," Journal of Vibration and Control, vol. 53, no. 5, pp. 573-579, 2013.

[17] I. G. Raftoyiannis, "Parametric resonance of steel bridges pylons due to periodic traffic loads," Archive of Applied Mechanics, vol. 82, no. 10-11, pp. 1601-1611, 2012. 

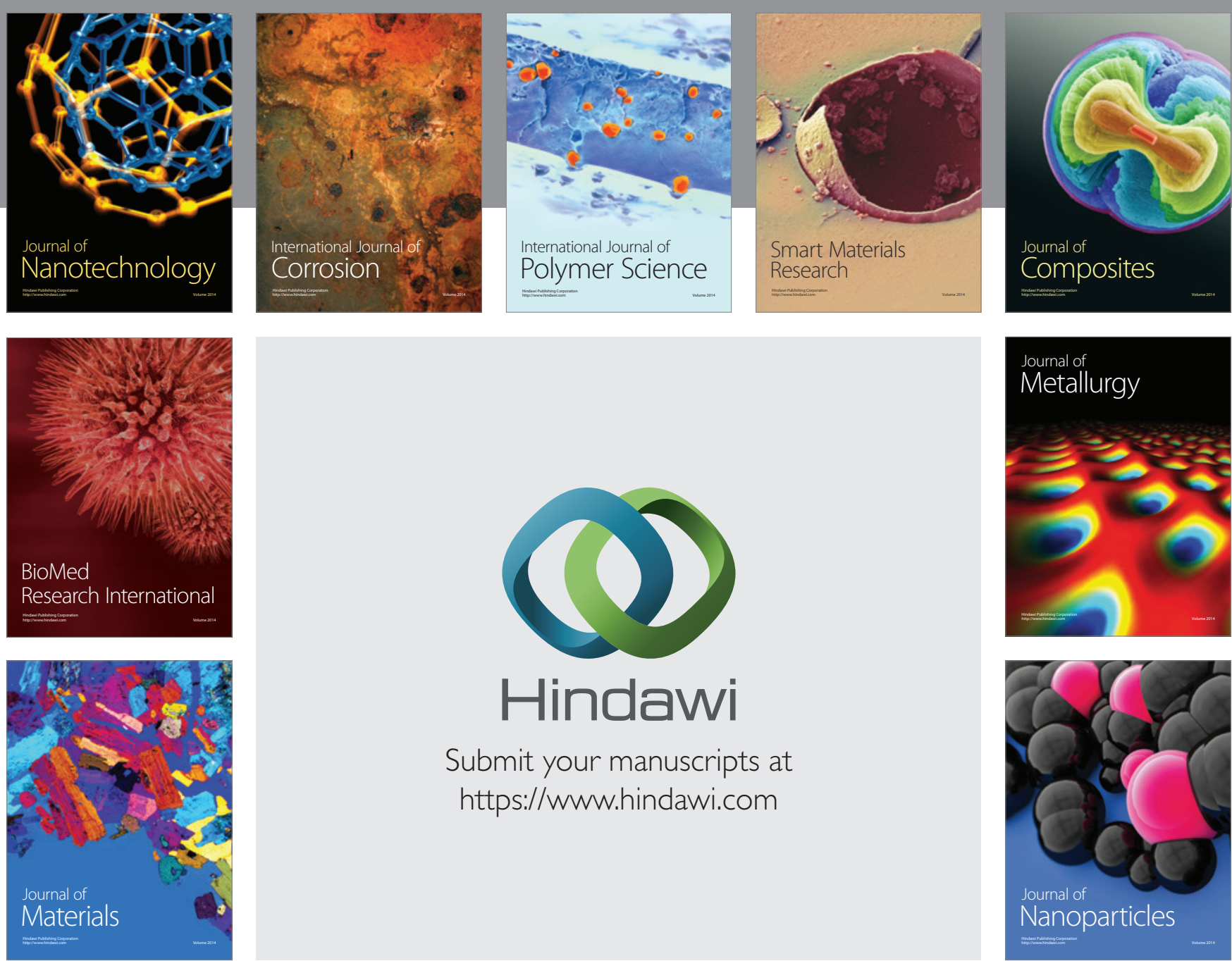

\section{Hindawi}

Submit your manuscripts at

https://www.hindawi.com

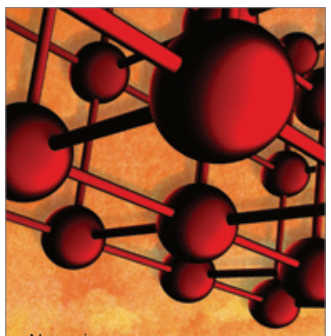

Materials Science and Engineering
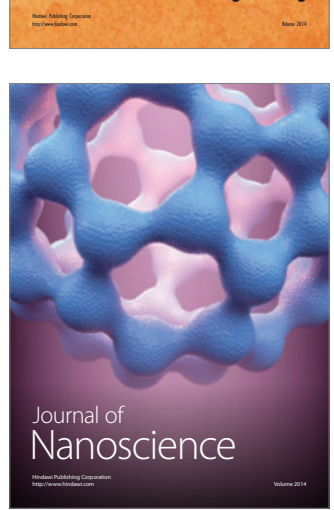
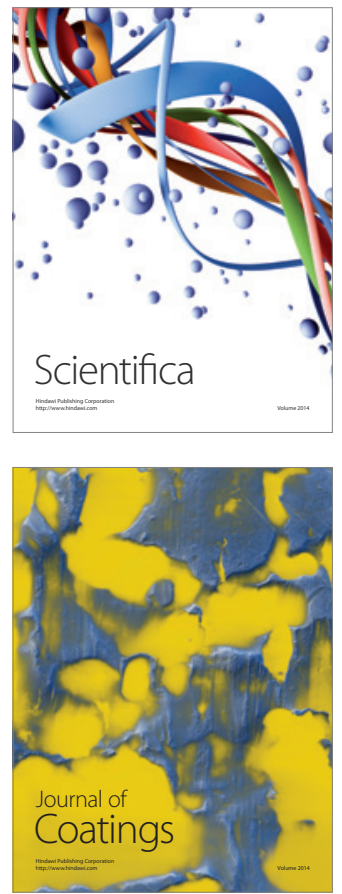
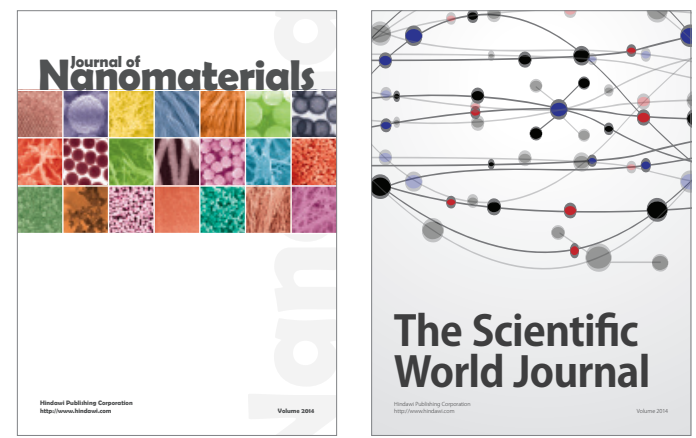

The Scientific World Journal
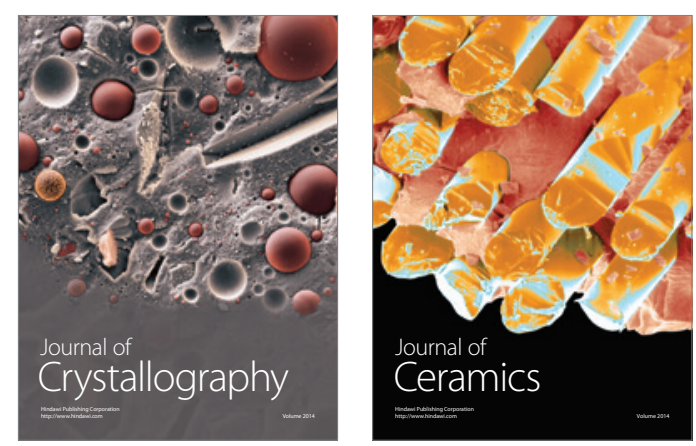
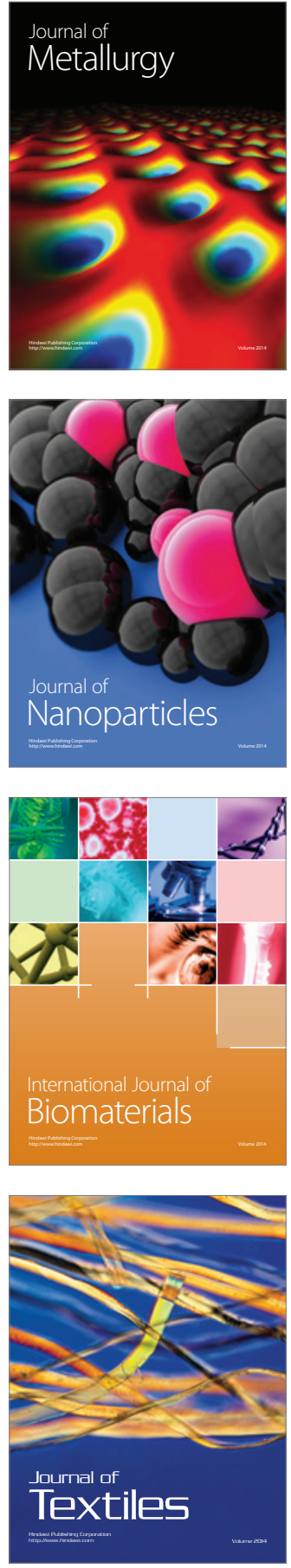